Fifth International Conference on Sustainable Construction Materials and Technologies. http://www.claisse.info/Proceedings.htm

\title{
IMPACT OF SUSTAINABLE BUILDING DESIGN ON OCCUPANT EXPERIENCE: A HUMAN CENTERED APPROACH
}

\author{
Antony Darby ${ }^{1}$, Sukumar Natarajan ${ }^{1}$, David Coley ${ }^{1}$, Dan Maskell ${ }^{1}$, Ian Walker ${ }^{2}$, \\ James Brownjohn ${ }^{3}$
}

${ }^{1}$ Department of Architecture and Civil Engineering, University of Bath, Bath, UK

${ }^{2}$ Department of Psychology, University of Bath, Bath, UK

${ }^{3}$ College of Engineering, Mathematics and Physical Sciences, University of Exeter, Exeter, UK

\begin{abstract}
All too often sustainability in building design is decoupled from user experience. This paper discusses how user comfort, well-being and performance should be put at the centre of the design process and how work beginning at Bath and Exeter, using the newly built VSimulators facility, is helping shape this. From a structural perspective, the drive to reduce material use, the use of more sustainable materials (e.g. CLT) and the desire to improve structural efficiency can lead to building designs which are not governed by ultimate limit state criteria, but rather by serviceability limit states. Reduced stiffness, in particular, leads to undesirable floor vibrations and/or sway in tall buildings. The question arises as to what is an acceptable level of vibration or motion. While it is a relatively simple task to define perception thresholds, acceptability is an altogether more complex problem.

Subjective measures of acceptability vary significantly from person to person and is situation, context and task dependent. What's more, it is not just vibrations that affect the acceptability of the indoor building environment; temperature, humidity, air quality, lighting, noise, even smell, all have an influence on whether a building is fit for purpose. Sustainable construction practices, such as passive house design and air tightness, can lead to poor environmental conditions (e.g. increased concentrations of VOCs) unless we consider the impact on the occupants. Thus, the work being carried out using the VSimulator facility aims to understand these complex interactions between structural, environmental and human factors, using a multidisciplinary approach involving psychology, physiology, engineering, building physics and health. The challenges this issue poses and the unique facilities developed to address these challenges are described in this paper.
\end{abstract}


Keywords: Vsimulator, sustainability, building environment

\section{INTRODUCTION}

Many engineers and designers of buildings inevitably think of sustainability as always being a good thing. And, of course, it is when considered from the correct perspective. However, what often happens is that the perspective is very narrow looking only at, for example, reducing material usage, or improving energy consumption. But there is a need to take a more holistic approach to design - one which considers unintended consequences for the users of a building. As an example, the use of CLT timber for constructing multi-storey buildings is gathering pace, due to the perceived sustainability of timber products (Reynolds et al. 2011). While strength of these structures is not in doubt, these materials are significantly less stiff than the more conventional steel and concreted counterparts. This, then, leads to the possibility of structures which are significantly more flexible (Abeysekera and MálagaChuquitaype, 2015) and therefore have a propensity to move under the action of both wind loading and the humans moving around the building. So, there is a transition from ultimate limit state driven designs to a serviceability limit state driven designs. The problem now becomes what is an acceptable level of movement, which is something for which there is very little agreement (Kwok et al. 2009), and can these levels be achieved without us changing our design practices.

As another example, the idea of reducing energy required to heat a building through, perhaps, Passivhaus design is clearly an important aim in order to reduce greenhouse gas emissions. However, this is often achieved by making buildings highly insulated with high levels of air tightness (McLeod et al. 2013). This has led to buildings which overheat and are therefore uncomfortable for occupiers. This is exacerbated by use of unreliable models of human behavior and their thermal interaction with their environment in the design of a building environment and the predictions of performance. What's more, there are potential issues surrounding build-up of volatile organic compounds (VOC's) from furniture, carpet, fittings, cleaning sprays and liquids, etc, all of which can have undesirable health implications for building occupants.

The clear common problem with both situations is that we do not fully know:

1. How humans experience, interact with, and influence these effects.

2. What are acceptable levels and how do other environmental factors influence this acceptability.

3. How we can prevent or mitigate against these unintended consequences to meet acceptable levels.

The Universities of Bath and Exeter have been awarded funding to develop and build two pieces of equipment which will help us investigate and answer these questions. Together, the resulting facilities are known as VSimulators and they combine motion, vibration and environmental control with virtual reality to simulate a range of built 
environments. These facilities are specifically aimed at examining and understanding human response to building environmental factors and developing rational acceptance criteria which are context dependent. They can also be used for testing materials and systems which help mitigate problems associated with different internal environments and for developing non-structural interventions which can offset the unintended consequences of sustainable construction practices. This will contribute to the next generation of sustainable buildings by providing evidence backed design requirements which directly account for user experience, be that comfort, health, well-being or productivity.

\section{VSIMULATORS HUMAN TESTING FACILITY}

The VSimulators facility at the Universities of Exeter and Bath provides unique simulation capability that addresses critical issues of human engagement with the built environment. The two sites share resources and a common mission: to enable fundamental and applied research into human interactions in and with buildings and structures with the potential to transform research methods across a wide range of disciplines. Between them the two simulators provide motion capabilities in all axes across the widest range of movement and frequency content. However, unlike any other dynamic testing facility, VSimulators combines the effects of the full range of environmental conditions, motion, audio and visual cues in real-world settings on humans whose reactions and interactions are recorded by a full spectrum of instrumentation. By combining multi-disciplinary expertise across structural engineering, material science, virtual reality, health, physiology, psychology, building physics and environmental engineering, VSimulators has the potential to revolutionise research into how humans respond to environment and how we can manipulate environments in a sustainable way to make them more acceptable to users.

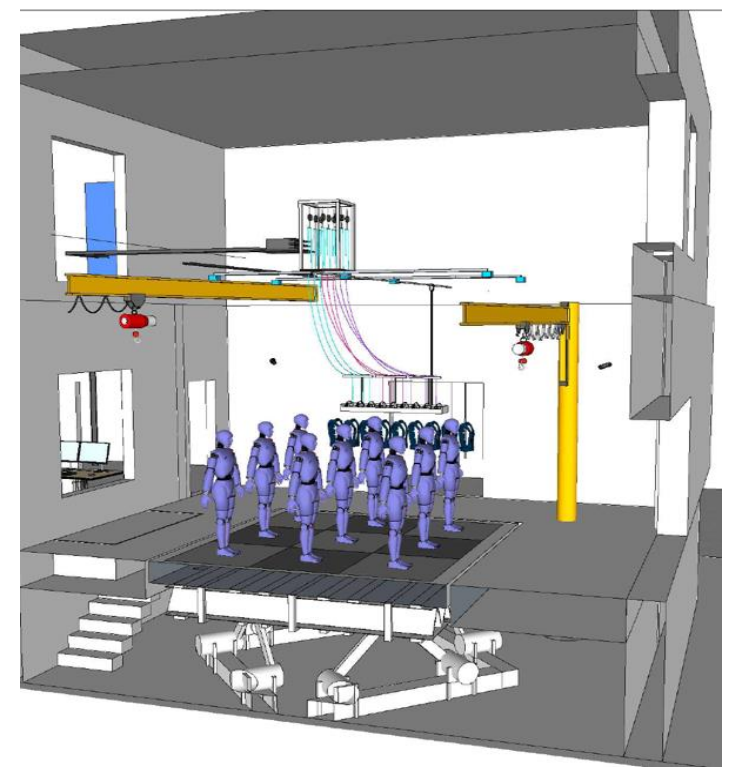

Figure 1: Exeter VSimulator equipment 
The Exeter equipment consists of a $4 \mathrm{mx} 4 \mathrm{~m}$ rigid platform driven by electric actuators arranged in a 'hexapod' arrangement allowing motion in all six translational and rotational axes (Figure 1). This is optimised for motion simulation in the range 0.5$40 \mathrm{~Hz}$, with hexapod positioning precision of the order of microns (see Figure 2). It uses state of the art VR with head mounted displays (HMDs), providing for realistic audio-visual simulation of built environments.

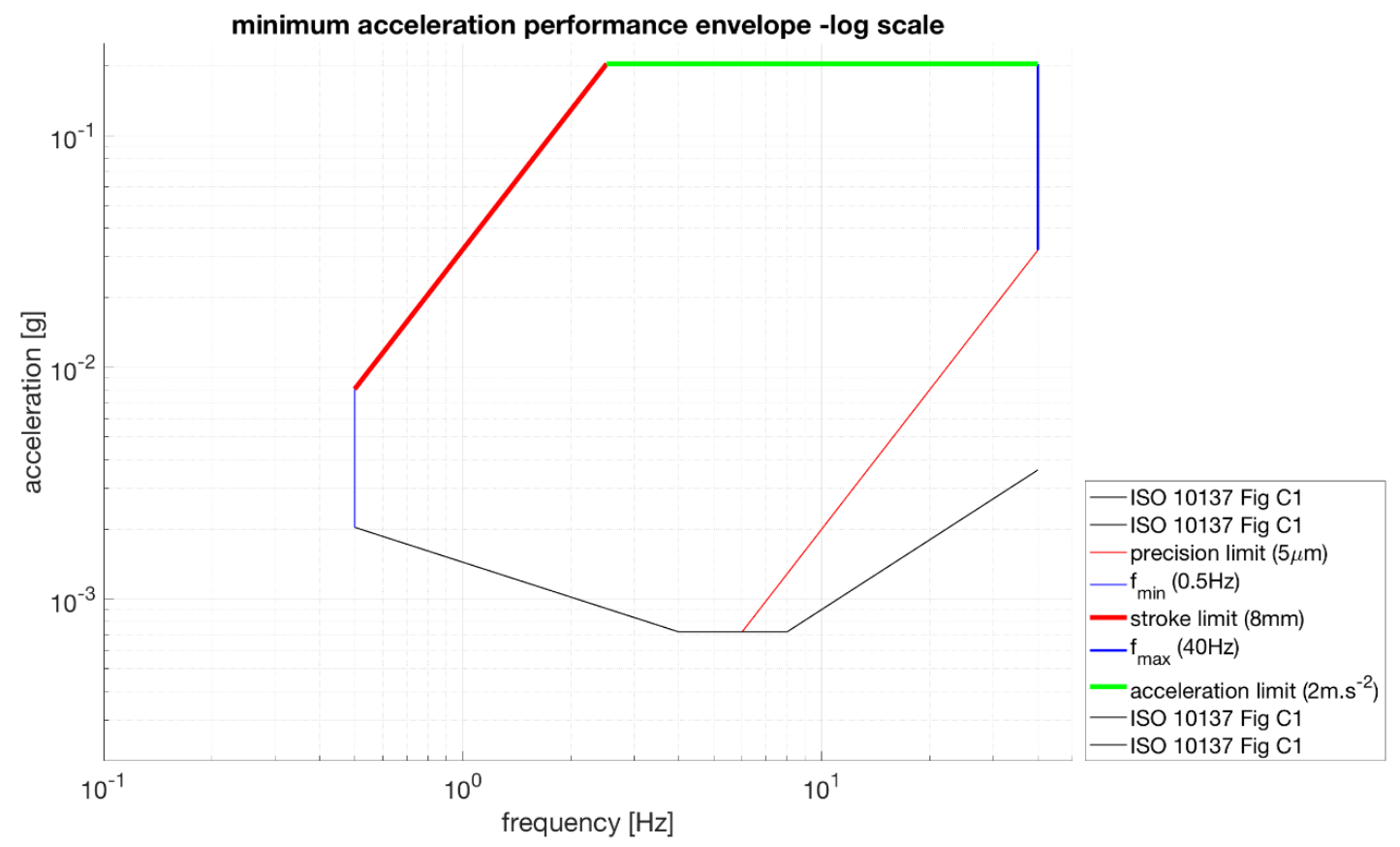

Figure 3: Exeter equipment motion envelope (six-degrees-of-freedom)

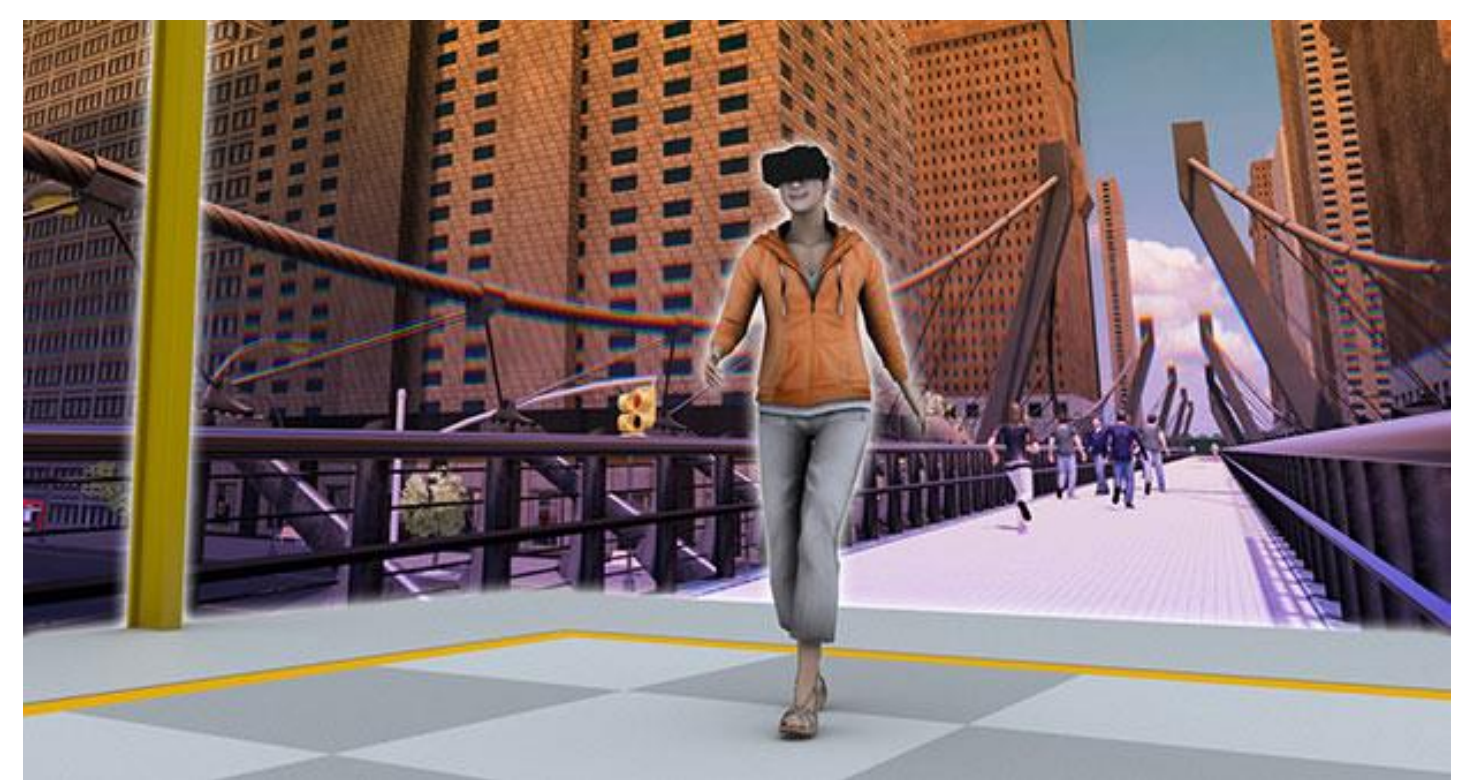

Figure 2 Exeter equipment Virtual Reality scenario - endless bridge 
The floor is covered with an array of multi-axis force plates to recover ground reaction forces generated by users. The open platform is in a controlled laboratory environment which enables occupant motion capture using both optical and inertial systems. Removable multi-user treadmills and fans can also be used to enhance simulation of open space movement (Figure 3). The platform provides capability to study human and motion factors on footbridges, floors, grandstands etc. as well as a host of applications in healthcare and rehabilitation (trips, falls, assisted mobility), movement science (posture, balance and locomotion), human-human and human-structure interaction in moving built environments.

The Bath equipment (Figure 4) is a building sway simulator but also uniquely features a climate controlled chamber with precise environmental control, together with extensive occupant physiological monitoring. It can mimic biaxial (horizontal) building sway up to $0.8 \mathrm{~m}$ at low frequencies $(0.025-6 \mathrm{~Hz})$ and accelerations from below $0.004 \mathrm{~g}$ (below the threshold of perception) and up to at least $0.06 \mathrm{~g}$ (above which walking becomes difficult), as shown in the motion envelope of Figure 5. This allows simulation of the full range of horizontal motions typical of medium to tall buildings, when subject to wind loading.

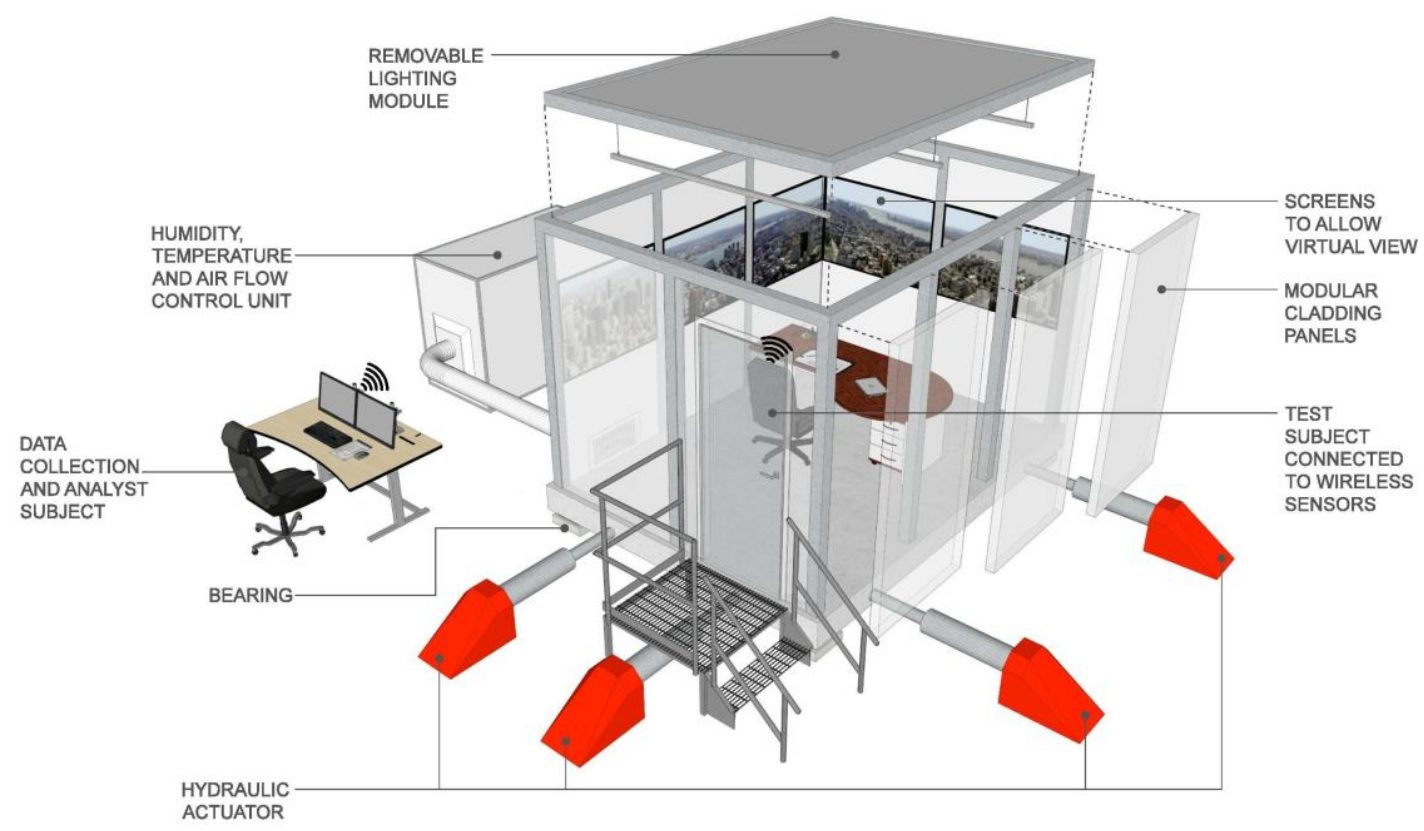

Figure 4: Schematic of Bath VSimulator

The climate chamber, which sits on top of the motion platform, is reconfigurable as an office, hotel, sitting room, hospital room, etc. Optical 3-D virtual reality environments are projected on the walls of the chamber to give a sense of space, and surrounding beyond the confines of the chamber (Figure 6), as well as virtual windows with views across a cityscape to give a sense of external conditions and height (Figure 7). 


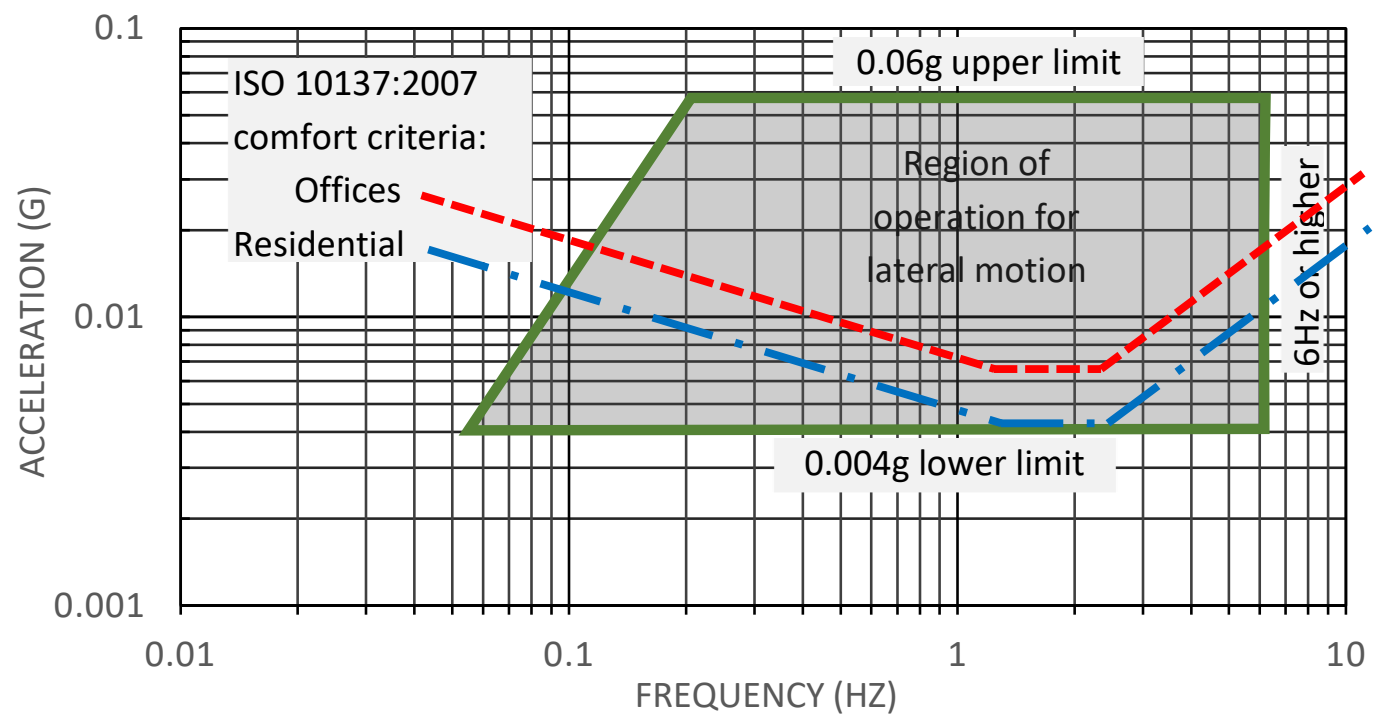

Figure 5: Bath simulator facility lateral motion envelope

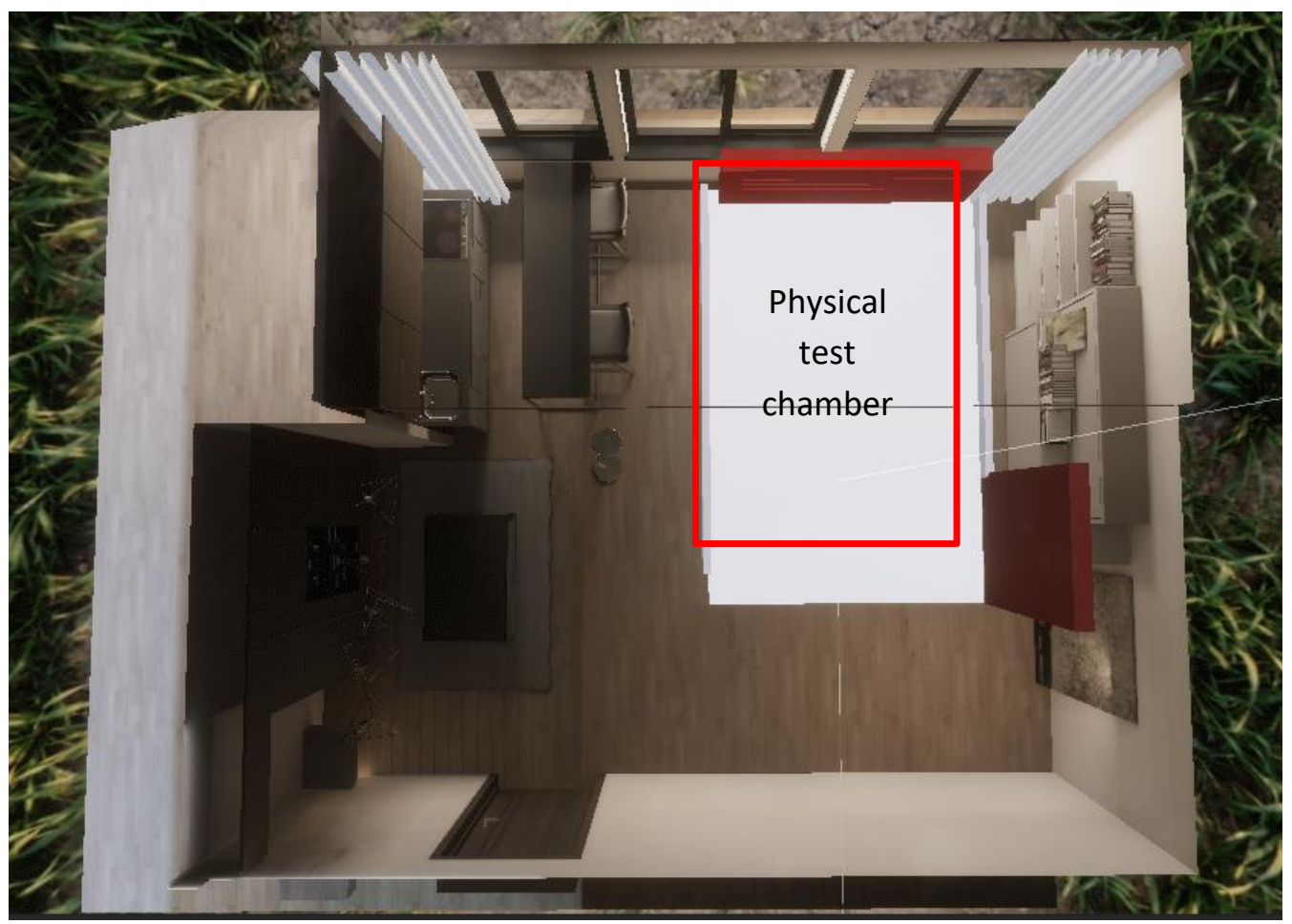

Figure 6: Virtual apartment indicating position of real test chamber

These external actors (motion, view, surroundings) can be varied and manipulated to explore their effects on occupants and their influence on perceived acceptability of motion and environmental factors. Internal environmental factors such as lighting intensity and colour temperature, air and radiant temperature, humidity, smell, noise, 
air flow and air quality can all be manipulated (with or without the physical motion and VR simulation) in the ranges shown in Table 1

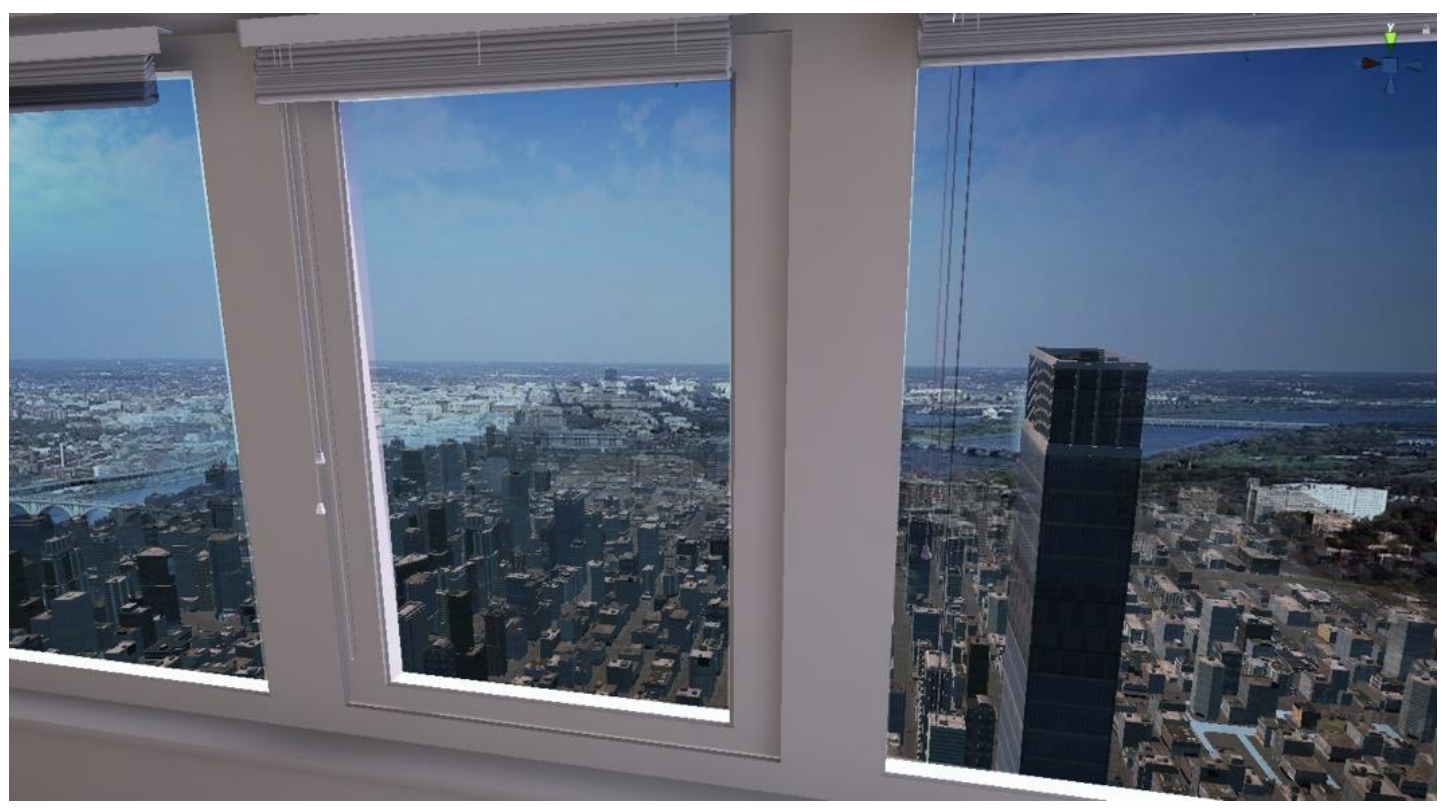

Figure7: Virtual windows and external views

Panels constructed using novel materials or construction can also be introduced internally to study their effect on internal environmental conditions, such as moisture buffering, or VOC regulation. All these controllable systems allow interactions among the various stimuli which affect building occupant health, comfort and productivity to be studied. In order to establish objective, quantifiable measures that represent the impact of the environment on users (such as increased stress, reduced concentration, tiredness) heat rate, blood pressure, galvanic skin reaction, eye movement, blink rate, electro-cardiogram and blood-oxygen levels can be measured using wireless sensors, with a low physical impact on occupants. By coupling the quantifiable measurements from these physiological sensors with the imposed environmental stimuli and with the more subjective responses of human test subjects through psychological testing protocols, it is possible to establish causal links between disturbing environmental inputs and their perceived acceptability. The equipment therefore allows researchers, for the first time, to study human response to the interaction between motion and/or environmental stimuli under fully controlled laboratory conditions yet in the context of real world surroundings, provided by virtual reality. This will give a profound insight into the structural and environmental requirements for serviceable buildings. 
Table 1. Bath VSimulator Human rated environmental chamber specification

\begin{tabular}{|c|c|c|c|c|}
\hline Controllable parameters & Min & Max & Tolerance & $\begin{array}{l}\text { Rate of } \\
\text { change }\end{array}$ \\
\hline Air Temperature (Degrees C) & 15 & 40 & 0.5 & 10 / half hour \\
\hline Radiant Temperature (Degrees C) & 15 & 40 & 0.5 & $\begin{array}{l}10 / \text { half hour } \\
5 \% \text { / half }\end{array}$ \\
\hline Humidity ( $\mathrm{RH}$ over the whole range of $\mathrm{T}$ ) & $20 \%$ & $80 \%$ & $5 \%$ & hour \\
\hline $\begin{array}{r}\text { Above three achieved at: } \\
\text { thermal Load (W) } \\
\text { moisture load }(\mathrm{kg} / \mathrm{hr})\end{array}$ & & $\begin{array}{l}200 \\
5\end{array}$ & & \\
\hline Air Flow (m/s) & 0.05 & 1.5 & 0.05 & $\begin{array}{l}0.05 / 15 \\
\text { mins }\end{array}$ \\
\hline $\begin{array}{l}\text { Fresh air requirement }(\mathrm{l} / \mathrm{s} / \mathrm{p}) \text { (linked to } \\
\mathrm{CO} 2)\end{array}$ & 1 & 10 & 0.5 & $1 / 15$ mins \\
\hline $\begin{array}{l}\text { input simulated sound SPL ( } L p d B) \\
\text { Ability to adjust reverberation time }\end{array}$ & 0 & 100 & 10 & \\
\hline $\begin{array}{l}\text { Ability to adjust reverberation time } \\
\text { (seconds) }\end{array}$ & 0.2 & 1 & 0.1 & $\begin{array}{l}\text { steps of } 0.1 \\
\text { ability to }\end{array}$ \\
\hline Horizontal Illuminance (lux) & 50 & 2000 & 25 & $\begin{array}{l}\text { change } \\
\text { change in }\end{array}$ \\
\hline Correlated Colour Temperature range (K) & 2000 & 6500 & 250 & steps of $500 \mathrm{k}$ \\
\hline
\end{tabular}

\section{CURRENT RESEARCH USING BATH VSIMULATOR}

As the Bath VSimulator equipment is being commissioned, there are two research projects underway which allow us to explore important research challenges awhile at the same time explore the capabilities of the equipment. Thus the first project project relies on the motion simulation capability while the second project examines the effect of indoor environmental quality. Both involve the physiological and psychological testing of volunteers from across a range of gender, age and socioeconomic backgrounds

\section{Sopite Syndrome due to Persistent Low Frequency Sway}

As stated earlier, the use of certain more sustainable structural materials such as timber products, has the potential for low structural stiffness resulting in significant lateral sway due to wind loads in even relatively low rise buildings. This is exacerbated as buildings get ever taller in order to make better use of expensive real estate in cities around the world. The problem designers have is that there very little understanding of what level of motion is acceptable; current guidance that is available is based entirely on perception thresholds. Whether or not building motion can be perceived is almost entirely irrelevant since acceptability of the environment that building occupants work and live in involves many factors; it is context dependent. So, for example, as extreme cases, those working in a very active environment, such as a dance studio would accept very different levels of vibration than those carrying out precision electronics work. Of course, most of us work somewhere in between these 
extremes, and do a variety of activities during the day, but the principle is that what people do, the environment they do it in, and their expectations of that environment influence whether or not any motion is acceptable. Moreover outside of work and leisure, there is an assumption that those relaxing at home can tolerate (or, more accurately, perceive) much lower levels of vibration or sway than those in an office environment (see Figure 5). However, there is no experimental basis or evidence for this assumption and, indeed it is quite possible that the reverse is true in many circumstances (Lamb, S. and Kwok, KCS, 2017a).

Returning to the assumption that perception of motion is somehow related to acceptability, there is now evidence that, motion below the perception threshold can still lead to negative changes in physiology and behavior (Lamb, S. and Kwok 2017b), $\mathrm{KCS}$,. In particular this is seen in the form of 'sopite syndrome' which correspond to feelings of drowsiness, lethargy, low mood and lack of energy. Sopite syndrome is the effect that is typically made good use of in rocking babies to sleep, but is generally detrimental in a busy and productive work situation. The current study underway is looking at sopite syndrome under the action of persistent low level sway motion. By specifically aiming this research at acceptability criteria, rather than perception criteria, a robust framework will be provided for assessing and designing buildings which are fit for purpose. Most previous work associated with tall building motion has been carried out using artificial, constant frequency, constant amplitude sinusoidal motion. While this may well be a valid approach for defining perception thresholds, it is not well suited to examining acceptance levels. Therefore, the work carried out in this study will use realistic random narrow band motion representative of actual measured motion of buildings.

To examine sopite syndrome the first stage is to define how long and under what circumstances the onset of sopite syndrome occurs in most building occupiers. Identifying the onset of sopite syndrome is difficult since it involves symptoms which are hard to identify physiologically. Therefore proxies are used to identify changes in state. For example, increases in frequency of blink rate, increases in length of blink or low blood oxygen levels can all indicate tiredness associated with sopite syndrome, but this needs to be correlated with psychological testing and subjective "vote" type questionnaires if it is to be directly attributed to sopite syndrome, rather than simply general tiredness. There is some contradiction in the literature about whether work performance is affected by sway motion. This may be due to different types, frequencies and magnitudes of acceleration associated with the applied motion as well as the different task types being asked of subjects. The current study therefore involves correlating physiological signs of sopite syndrome with observed changes work performance for a range of tasks (such as text typing, proof reading, memory and perception tasks) which are related to different aspects of work behavior, such as cognition, memory, physical dexterity, etc.

The question then arises as to how sopite syndrome can be prevented by either appropriate building design or other mitigating solutions. Even if the building motion itself cannot be changed are there other things that can be done to offset the symptoms 
of sopite syndrome such as encourage physical activity, increase lighting levels, lower room temperatures or improve air quality? This then starts to be influenced by another problem within our modern, sustainable, energy efficient buildings; they are often overheated and poorly ventilated with poor air quality. This merely exacerbates the problem of sopite syndrome. Thus there is a need to consider the mix of environmental and physical conditions that lead to these undesirable effects and define exactly how much each factor influences human well-being and work performance.

\section{Indoor Air Quality (IAQ) and Productivity}

Statutory regulation for the decarbonisation of the built environment through improved energy efficiency is, as discussed earlier, leading to an unintended consequence of a deterioration in indoor environmental quality. Considering that people within industrialised countries spend approximately $90 \%$ of their time indoors, there is significant potential for exposure to poor environments. This impacts occupants' health and wellbeing and can result in a reduction of productivity or work performance, thus having both a societal and economic impact (World Green Building Council, 2015). However, evidence which demonstrates and quantifies the relationship between the indoor environment and occupant wellbeing and productivity (rather than comfort which up to now has been the main research focus) is limited. The state of the art, with respect to the indoor environments is largely through the monitoring of various parameters and subsequently inferring the potential levels of impact from secondary evidence. The Bath VSimulator equipment, being used for this project, allows careful control of the indoor environment and both direct and indirect human testing. This allows various human performance indicators to be monitored, including cognitive function and response rates in addition to physiological measurements which can then compared and correlated against changes in temperature, humidity and ventilation. This will enable the complex relationship of the indoor environment and its impact on human wellbeing and productivity to be understood. This will provide the required robust data set to justify future design criteria and further research in a range of disciplines. By understanding these environmental and air quality issues a new approach to the design of indoor environments based on wellbeing or productivity can be developed, rather than simply trying to achieve acceptable levels of comfort (and, even this criteria is often failed to be met in modern sustainable environments deigned to save energy rather than focus on users). This research will subsequently enable future developments for providing active and passive solutions of controlling the indoor environment to meet the well-being criteria.

Just like the dichotomy between motion perception and motion acceptability, environmental comfort and well-being are two different concepts that could not be used interchangeably. The first is entirely subjective and to do with the individual preference for a particular environmental state, such as thermal comfort (which humans are well adapted to sense, unlike something like relative humidity, which humans find much more difficult to judge, yet still has an impact). Well-being on the other hand is a multidimensional concept and there is little agreement for how it should be defined or what it encompasses. At one extreme it can relate to physical and mental health of a person. A range of different illnesses are associated with high or low relative humidity and air 
pollutants, with concentration levels empirically associated with temperature and levels of carbon dioxide. These aspects are more quantitative in nature but, technically and ethically, challenging to study. At the other extreme well-being can relate to a more abstract psychological state, such as 'happiness' or 'satisfaction', which are more nebulous and subjective in nature. VSimulators has some of the means to explore these definitions of well-being, but would require longitudinal studies in the real-world environment in order to properly calibrate results and findings.

The effect of indoor environment on productivity and work performance on the other hand is more quantitative in nature and this is the focus of this current project. By using some of the same physiological and psychological test protocols described for the study of sopite syndrome, the links between changes in the ability to satisfactorily do various work tasks (both in terms of efficiency and effectiveness) are being explored. Most previous indoor environmental research has focused on the effect of environmental factors upon human cognitive performance but this is not the only important issue that should be considered. The problem is that it is possible that some occupants maintain the same, or better, cognitive performance in more difficult environmental situations but with higher cognitive load which can lead to increase in mental stress (Zhang et al, 2017). VSimulators allows indicators of stress to be measured at the same time as work performance is considered in relation to the environmental quality. For the current research, the environmental focus is on indoor air quality (temperature, humidity and ventilation), although it is recognised that other micro-environment factors such as lighting, noise level, furniture comfort, etc), also greatly influence productivity This will be the subject of future research studies.

\section{CONCLUSIONS}

This paper describes some of the unintended negative consequences of current sustainability driven building design practices, both structural and environmental. It is imperative that we understand how these environmental factors affect the wellbeing, health, comfort and productivity of building users. With this in mind a new experimental facility, VSimulators, has been developed to allow us to investigate the various interactions between environmental and physical conditions on the users of buildings. The output of research using the facility will help develop new user centered sustainable design focus. Buildings need to be designed with context dependency and multi-objective human acceptability in mind rather than merely attempting to meet standardised criteria based on a single objective, such as energy efficiency, which ignores impact on users. This will require appropriate use of materials and construction technologies to meet the challenging requirements. 


\section{REFERENCES}

Reynolds, T., Chang, W. and Harris, R. (2011). "Wind-induced vibration of tall timber buildings." Paper presented at IASS Annual Symposium: IABSE-IASS 2011: Taller, Longer, Lighter, London, UK United Kingdom.

Abeysekera I. and Málaga-Chuquitaype C. (2015). "Dynamic response of tall timber buildings", Earthquake Risk and Engineering towards a Resilient World, 9-10 July, Cambridge UK.

McLeod, R.S., Hopfe, C.J., Kwan, A. (2013). “An investigation into future performance and overheating risks in passivhaus dwellings." Building and Environment 70.

Kwok, K.C.S., Hitchcock P.A. and Burton, M. (2009). "Perception of vibration and occupant comfort in wind-excited tall buildings." Journal of Wind Engineering and Industrial Aerodynamics, 97(7), 368-380.

Lamb, S. and Kwok, K.C.S. (2017a). "The Fundamental human response to wind induced building motion." Journal of Wind Engineering and Industrial Aerodynamics, 165, 79-85.

Lamb, S. and Kwok, K.C.S. (2017b) "Sopite syndrome in wind-excited buildings: productivity and wellbeing impacts." Journal of Building Research and Information, $5(3)$.

World Green Building Council. (2015), Health, Wellbeing \& Productivity in Offices. Zhang, F., Haddad, S., Nakisa, B., Naim Rastgoo, N., Candido, C., Tjondronegoro, D., de Dear, R. (2017). "The effects of higher temperature set points during summer on office workers' cognitive load and thermal comfort." Building and Environment 123 176-188. 